\title{
NAPSB as a Predictive Marker for Prognosis and Therapy Associated with an Immuno-Hot Tumor Microenvironment in Hepatocellular Carcinoma
}

Qiu Zhao ( $\square$ qiuzhao@whu.edu.cn)

Zhongnan Hospital of Wuhan University

\section{Yu-Mei Ning}

Zhongnan Hospital of Wuhan University

Kun Lin

Zhongnan Hospital of Wuhan University

Xiao-Ping Liu

Zhongnan Hospital of Wuhan University

Yang Ding

Zhongnan Hospital of Wuhan University

Xiang Jiang

Zhongnan Hospital of Wuhan University

Zhang Zhang

Zhongnan Hospital of Wuhan University

Yu-Ting Xuan

Zhongnan Hospital of Wuhan University

Lan Liu

Zhongnan Hospital of Wuhan University

\section{Fan Wang}

Zhongnan Hospital of Wuhan University

Hai-Zhou Wang

Zhongnan Hospital of Wuhan University

Jun Fang

Zhongnan Hospital of Wuhan University

\section{Research Article}

Keywords: NAPSB, Hepatocellular carcinoma, Tumor microenvironment, Immunotherapy, Chemotherapy

Posted Date: March 7th, 2022

DOI: https://doi.org/10.21203/rs.3.rs-1417814/v1 
License: (c) (i) This work is licensed under a Creative Commons Attribution 4.0 International License. Read Full License 


\section{Abstract}

Background: Napsin B Aspartic Peptidase, Pseudogene (NAPSB) was associated with CD4+ T cell infiltration in pancreatic ductal adenocarcinoma. But the biological role of NAPSB in hepatocellular carcinoma (HCC) remains to be determined.

Methods: The expression of NAPSB in pan-cancer and HCC as well as its clinicopathological association were analyzed using data from several public datasets. qRT-PCR was used to verify the relative expression of NAPSB in HCC using Zhongnan cohort. Kaplan-Meier analyses, univariate and multivariate Cox regression were conducted to determine the predictive value of NAPSB on HCC prognosis. Then enrichment analyses were performed to identify the possible biological functions of NAPSB.

Subsequently, the immunological characteristics of NAPSB in the HCC tumor microenvironment (TME) were demonstrated comprehensively. The role of NAPSB in predicting hot tumors and its impact on immunotherapy and chemotherapy responses was also analyzed.

Results: NAPSB was downregulated in HCC and high NAPSB expression showed an improved survival outcome. Enrichment analyses showed that NAPSB was related to immune activation. NAPSB was positively correlated with immunomodulators, tumor-infiltrating immune cells (TIICs), T cell inflamed score, and cancer immunity cycles and highly expressed in immuno-hot tumors. High expression of NAPSB was sensitive to immunotherapy and chemotherapy, possibly due to its association with pyroptosis, apoptosis and necrosis (PANoptosis).

Conclusions: NAPSB was correlated with an immuno-hot and inflamed TME and tumor cell death. It can be utilized as a promising predictive marker for prognosis and therapy in HCC.

\section{Introduction}

Hepatocellular carcinoma (HCC) is the sixth most frequent malignancy worldwide and the third leading cause of cancer-related deaths, accounting for almost $90 \%$ of primary liver cancers [1]. Systemic treatments are the important options for HCC patients as a large portion of HCC patients have already reached advanced stage when they first diagnosed and lost the opportunity of surgery [2]. In addition, emerging immunotherapies involving the use of immune checkpoint inhibitors (ICls) are currently the focus of research in many advanced cancers [3-6]. However, both systemic chemotherapy and immune checkpoint therapy have limitations of drug resistance or response in only some patients [7-9]. Therefore, identifying new biomarkers is urgent and benefits for the individual therapy.

In HCC, the tumor microenvironment (TME) composed of cancer cells, immune cells and extracellular matrix has an immunosuppressive effect, promoting immune tolerance and avoidance [10]. However, recent studies have shown that abundant infiltration of CD8+, CD4+, regulatory $T$ cells and dendritic cells (DCs) can shape an inflamed TME to play a potential anticancer role and influence the efficacy of ICls [11-13]. Based on the characteristics of the TME, tumors can be divided into hot and cold tumors. Hot tumors are characterized by T-cell infiltration, molecular characteristics of immune activation and 
responsive to cancer immunotherapy, while cold tumors are characterized by the opposite [14]. In HCC, such molecules and pathways, including $\beta$-catenin, PPARG (peroxisome proliferator activated receptor gamma)-network, and IDH1 (isocitrate dehydrogenase 1) pathways, have been shown to promote the formation of a non-inflamed TME [15-17], while IFN- (interferon-gamma), APM (antigen processing and presenting machinery), EGFR (Epidermal growth factor receptor), and hypoxia pathways contributed to shape an inflamed TME [18-21].

Pseudogenes were considered to be "junk genes" for many years because of its abrogated transcription due to harbor mutations [22]. However, over the past decade, non-coding RNAs including pseudogenes, long non-coding RNAs (IncRNAs), and microRNAs have been demonstrated to play key roles in TME [23, 24]. NAPSB is a pseudogene that had been identified to be associated with the infiltration of CD4 + T immune cells in pancreatic ductal adenocarcinoma (PDAC) [25]. In the study of Anirban et.al [26], NAPSB was highly expressed and identified as a hub gene in pre-eclampsia with systemic inflammatory response. In HCC, NAPSB was found to be downregulated [27] but its biological role has not been elucidated. Thus in this study, the potential biological functions of NAPSB were comprehensively explored in HCC, including its differential expression, prognosis value and immunological role. We also reported that high NAPSB expression was related to an immuno-hot TME and sensitive to immunotherapy/chemotherapy possibly on account of affecting pyroptosis, apoptosis and necrosis (PANoptosis) in HCC.

\section{Materials And Methods}

\section{Public data collection}

TIMER database (https://cistrome.shinyapps.io/timer/) was used to analyze expression levels of NAPSB in pan-cancer. HCC patients with the transcriptomic RNA-sequencing data (log2 $(F P K M+1)$ value) of The Cancer Genome Atlas (TCGA) datasets were obtained from the online data portal UCSC Xena (https://xenabrowser.net/datapages/). Patients with insufficient or missing clinical and prognosis information were excluded. Then the resting 369 TCGA-HCC samples were used. Meanwhile, LIRI-JP ( $n=231$ ) retrieved from International Cancer Genome Consortium (ICGC) database (https://icgc.org/) were chosen for primary external validation. In addition, we used multiple cohorts from Gene expression omnibus (GEO) (https://www.ncbi.nlm.nih.gov/geo/), including GSE55092, GSE54236, and GSE121248, to verify the relative expression of NAPSB in HCC and normal tissues.

Tow immunotherapy-related cohorts, GSE78220 and GSE91061 (melanoma), were downloaded from GEO database. GSE104580, a dataset of transcatheter arterial chemoembolization (TACE) for HCC patients was also downloaded.

\section{Tissue specimens acquisition}


13 hepatocellular carcinoma tissues and paired adjacent normal tissues were obtained from Zhongnan Hospital of Wuhan University between February 2021 and September 2021 with informed consents. The patients were diagnosed with hepatocellular carcinoma based on histopathological evaluation, and no local or systemic treatment was conducted before surgery. The protocols used in the study were approved by the Research Ethics Committee of Zhongnan Hospital of Wuhan University.

\section{Quantitative reverse transcription polymerase chain reaction (qRT-PCR) assays}

Total RNA was extracted from hepatocellular carcinoma and paired adjacent normal tissues using TRIZOL reagent (Invitrogen, Carlsbad, CA, USA). RNA quantity was determined by NanoDrop2000c (Thermo Scientific, Waltham, MA, USA). For qRT-PCR, $1 \mu \mathrm{g}$ RNA was reverse transcribed to CDNA using a Reverse Transcription Kit (Toyobo,Osaka, Japan). The qRT-PCR assays were conducted on LightCycler ${ }^{\circledR}$ 96. Target gene expression was normalized against GAPDH. The primer sequences were: NAPSBForward: CATCCAGTTTGCTCAGGGT; NAPSB-Reverse: TCGAAGACGGTCACATACGC; GAPDH-Forward: CCCCAGCAAGAGCACAAGAG; GAPDH-Reverse: GCACAGGGTACTTTATTGATGGTAC.

\section{Evaluate the prognostic value of NAPSB}

Kaplan-Meier (K-M) analyses, univariate and multivariate Cox regression were conducted to explore the influence of NAPSB on the survival of patients in HCC using R package "survminer" and "survival"to investigate the optimal cutoff of NAPSB expression and draw the $\mathrm{K}-\mathrm{M}$ survival curve, while the log-rank test was applied to estimate statistical significance. Overall survival (OS), disease-free interval (DFI), and progression-free interval ( $\mathrm{PFI}$ ) were evaluated ( $\mathrm{p}$-value $<0.05$ as significant).

\section{Analysis of NAPSB co-expressed genes and differential expressed genes}

Genes potentially positively co-expressed with NAPSB were predicted using R software. Those genes with a thresholds of $p$-value $<0.01$ and |Spearman's correlation $\mid \geq 0.45$ were selected for feather analysis. Patients were classified into two groups based on the median NAPSB expression. We screened differentially expressed genes (DEGs) between NAPSB subgroups using the "edgeR" package in the R software. An adjusted p-value $<0.05$ and $\mid$ log2 (fold change) | value $\geq 1.3$ was considered significant. We took the intersection of the co-expressed genes and the upregulated DEGs as the genes most related to NAPSB for further analysis.

\section{Biological function, pathway annotation, gene set enrichment analysis (GSEA) and gene set variation analysis}




\section{(GSVA)}

We conducted gene ontology (GO) and Kyoto Encyclopedia of Genes and Genomes (KEGG) pathway analyses to explore the possible biological function of the genes most related to NAPSB (mentioned above) via the R package "clusterprofiler", with a cutoff value of adjusted p-value $<0.05$. To investigate the difference in biological process terms between the NAPSB-high and NAPSB-low groups, GSEA was applied using the R package "clusterProfiler" and GSVA was applied using the R package "GSVA". The gene sets of "h.all.v7.4.symbols" and "c5.cp.kegg.v7.4.symbols" were downloaded from MSigDB for GSEA and GSVA respectively.

\section{Evaluation of relationship between NAPSB expression and the immunological characteristics of the TME}

Immunological characteristics of the TME in HCC include the expression of immunomodulators, infiltration level of TIICs and activity of the cancer immunity cycle. We first analyzed the correlation of NAPSB expression with immune-related genes, including major histocompatibility complex (MHC), immunomodulators, chemokines and receptors. A total 50 immunomodulators were collected from the study of Charoentong et al [28] (Supplementary Table 1). We applied the "Estimation of Stromal and Immune cells in Malignant Tumuors using Expression data" (ESTIMATE) algorithm to assess the immune scores, stromal scores, estimate scores, and tumor purity for each HCC sample [29]. Thereafter, we used several algorithms to comprehensively calculate the infiltration level of TIICs to avoid calculation errors: ssGSEA, TIMER [30], CIBERSORT [31], quanTIseq [32], EPIC [33], xCell [34], and MCP-counter [35]. We also identified the effector genes of TIICs from previous studies [36, 37] (Supplementary Table 2). The cancer immunity cycles included seven critical steps: cancer antigen release and presentation (Steps 1 and 2), anticancer immune priming and activation (Step3), immune cell trafficking (Step 4), immune cell infiltration into the TME (Step 5), T cell recognition of cancers (Step 6), and killing of cancer cells (Step 7) [38]. Moreover, we calculated the activities of these steps as described previously [39]. Finally, T cellinflamed gene expression profiles score was calculated as an average value of log2-scale normalized expression of the 18 signature genes [40].

\section{Unsupervised clustering}

Unsupervised clustering was implemented to classify HCC tissues into hot or cold tumors on the basis of hot tumor signature genes according to previous literature [41]. The number of clusters and their stability were determined by the consensus clustering algorithm. We used the "ConsensuClusterPlus" package to perform this algorithm and 1000 times repetitions were conducted for guaranteeing the stability of classification [42]. 


\section{Calculation of the enrichment scores of various gene signatures and prediction of immunotherapy response}

We analyzed the oncogenic pathways that were associated with targeted therapy, and immunotherapy responses according to previous research [36] (Supplementary Table 3). The enrichment scores of these signatures were calculated using the R package "GSVA" [43]. To analyze the efficacy of immunotherapy, tow immunotherapy-related cohorts, GSE78220 and GSE91061 (melanoma) were obtained.

\section{Prediction of chemotherapeutic response}

We downloaded the transcriptional expression data and drug response of more than 1000 cancer cell lines from Genomics of Drug Sensitivity in Cancer (GDSC, http://www. cancerrxgene.org/downloads) [44] and Cancer Therapeutics Response Portal (CTRP) [45] respectively. The Spearman correlation between the NAPSB of each cell line and half maximal inhibitory concentration (IC50) of each cell line to particular drugs was calculated. Correlations with adjusted p-value were filtered $<0.01$ as significant ones. In addition, GSE104580 was used to analyze the correlation between NAPSB expression and TACE response in HCC patients.

\section{Calculation of the enrichment scores of cell death gene sets}

We collected signatures of several forms of cell death, including pyroptosis, apoptosis, necroptosis, autophagy, and ferroptosis, from previous literatures [46-50] (Supplementary Table 4). The enrichment scores of these signatures were also calculated using the R package "GSVA" as mentioned above.

\section{Statistical analysis}

Statistical analyses were performed using R software (version 4.1.1) and SPSS software (version 25.0). Paired Student's t-test was performed to detect the differential expression of NAPSB in paired HCC and adjacent normal tissues. The significant difference in continuous variables between the two groups was measured by the Wilcoxon rank sum test, while categorical variables were compared using the chi-square test. Correlations between variables were explored using Pearson or Spearman coefficients. For all analyses, a two-paired p-value $<0.05$ was considered statistically significant if not noted. Statistical significance was defined as: ns, no significance; *, p-value $<0.05$; $* \star, p$-value $<0.01 ; * \star \star, p$-value $<0.001$; $\star \star \star \star$, $p$-value $<0.0001$.

\section{Results}




\section{Expression levels analysis and high NAPSB inferred a better prognosis for HCC}

NAPSB transcription levels in different human tumors were showed in Fig. 1a. Compared with adjacent normal tissues, expression of NAPSB in BLCA (bladder urothelial carcinoma), COAD (colon adenocarcinoma), LIHC (liver hepatocellular carcinoma), LUAD (lung adenocarcinoma), LUSC (lung squamous cell carcinoma) and READ (rectal adenocarcinoma) was significantly decreased but in BRCA (breast invasive carcinoma), ESCA (esophageal carcinoma), KICH (kidney chromosome) and KIRP (kidney renal papillary cell carcinoma) was significantly increased. For TCGA cohort, we analyzed paired samples by paired Student's t-test to verify the above results in HCC (Fig. 1b). To fully demonstrate this expression difference, we validated it with multiple datasets, including ICGC, GSE55092, GSE54236, and GSE121248, finding that NAPSB was indeed significantly decreased in HCC tissues (Fig. 1c).

Moreover, NAPSB expression was examined in 13 paired HCC and adjacent normal tissues of Zhongnan cohort from our hospital by RT-PCR and we obtained consistent results. (Fig. 1d).

The correlation between NAPSB and clinicopathologic characteristics for TCGA and ICGC cohorts were presented in Supplementary Table 5 and 6 . In addition, we investigated its survival value. K-M survival analysis showed that high expression of NAPSB was linked to better overall survival than its low expression (Fig. 1e) and more significantly associated with better disease-free survival (Fig. 1f) and progression-free survival (Fig. 1g). Its overall survival value was also verified in the ICGC cohort (Fig. 1h). Given that there are multiple factors affecting survival, univariate Cox regression analysis was performed using age, tumor stages, gender, and NAPSB expression as inputs; results showed that NAPSB expression were significantly associated with better DFI and PFI outcomes (Supplementary Fig. 1a) and multivariate Cox regression analysis further validated it (Supplementary Fig. 1b). Therefore, NAPSB expression was beneficial to overall survival and could serve as an independent predictor of disease-free survival and progression-free survival in HCC.

\section{Enrichment analyses inferred NAPSB was related to immune activation}

To investigate the potential biological function of NAPSB, we firstly performed a correlation analysis between NAPSB and other genes using RNA-seq data of HCC patients from the TCGA cohort. The results showed that there were 930 genes significantly associated with NAPSB ( $p$-value $<0.01$, ISpearman`s correlation $\mid \geq 0.45$; Supplementary Table 7). The correlation of NAPSB and the top 50 co-expressed genes was showed in Fig. 2a, which contained some immune-related molecules like CD48, CD37, IL6, HLA-DQA1, etc. Meanwhile, we analyzed the differentially expressed genes (DEGs) between the NAPSB high and low expression groups and found that there were 993 upregulated in the NAPSB-high group compared with the NAPSB-low group (adjusted $p$-value $<0.05$ and | log2 (fold change) | value $\geq 1.3$; Supplementary Table 8). The top 10 upregulated genes contained immune-related molecules, such as 
CD48, CD37, CCR5 (Fig. 2b). Both co-expressed genes and upregulated DEGs contained well-known molecules associated with immunity, suggesting that NAPSB may also be involved in immunity.

Thereafter, the intersection of co-expressed genes and upregulated DEGs including 476 common genes were obtained as the most closely related genes to NAPSB (Fig. 2c; Supplementary Table 9) and enrichment analyses were conducted in an attempt to reflect biological functions of NAPSB indirectly. The GO analysis showed that the common genes were enriched in processes such as $\mathrm{T}$ cell activation, leukocyte cell-cell adhesion, regulation of $T$ cell activation, and regulation of immune effector process, etc (Fig. 2d; Supplementary Table 10). The KEGG pathway analysis demonstrated that the common genes were associated with chemokine signaling pathway, Th17 cell differentiation and T cell receptor signaling pathway (Fig. 2e; Supplementary Table 11). Most biological functions and signaling pathways were immune-related, strongly implying that NAPSB may mediate the TME in HCC.

Even further, we conducted GSEA and GSVA between NAPSB high- and low- groups and also identified many significant pathways related to immunity (Fig. 2f, g; Supplementary Table 12,13) in the enrichment of MSigDB Collection (gene sets of "c5.cp.v7.4.symbols.gmt" and "h.all.v7.4.symbols"). These findings paralleled the above results.

\section{NAPSB shaped an immuno-hot and inflamed TME in HCC}

Given that the enrichment analyses indicated NAPSB was associated with immune activation in HCC, we subsequently comprehensively explored its immunological role using TCGA and ICGC cohorts. NAPSB was found to upregulated the expression of critical immunomodulators (including MHC, immunostimulator, chemokine, and receptor) (Fig. 3a), which may upregulate the activities of the cancerimmunity cycle subsequently. Additionally, ESTIMATE algorithm was applied to calculate the immune score, stromal score, estimated score and tumor purity. We found these scores were significantly increased NAPSB-high group (Fig. 3b). On the contrary, tumor purity was negatively correlated with the expression of NAPSB (Fig. 3c). Subsequently, TME immune cell infiltration was assessed by ssGSEA and a significant difference between NAPSB-high and NAPSB-low groups was observed. Almost all immune cells were significantly enriched in NAPSB-high group (Fig. 3d). Furthermore, we calculated the infiltration level of TIICs using other six independent algorithms (Fig. 3e). Consistent with the result of ssGSEA analysis, the infiltration levels of CD8+ T cells, CD4+ T cells, NK cells, B cells, DCs and macrophages were almost positively correlated with NAPSB in different algorithms. In particular, CD8+ T cells were positively correlated with NAPSB with statistical significance in all algorithms. In line with these, NAPSB was positively correlated with the marker genes of these six major types of immune cells (Fig. 3f). These results suggested NAPSB was associated with an inflamed TME. Even further, we observed the NAPSB expression positively correlated with the T cell inflamed score (TIS) established using IFN-y-related mRNA profiles [40] and all of genes within this signature (Fig. $3 \mathrm{~g}, \mathrm{~h}$ ), further confirming its roles in shaping a hot inflamed TME. These findings were all verified in ICGC cohort and obtained consistent results (Supplementary Fig. 2). 
Finally, we evaluated the correlation between NAPSB and seven steps of cancer-immunity cycle, which conceptualized the anti-cancer immune response [38]. Overall, In the NAPSB-high group, the activities associated with the majority of the steps in the cycle were notably upregulated (Fig. 3i), including the release of cancer cell antigens (Step 1), priming and activation (Step 3), trafficking of immune cells to tumors (Step 4), and infiltration of immune cells into tumors (Step 5). In summary, these data consistently indicated that high expression of NAPSB was to transform a non-inflamed TME into an immuno-hot and inflamed microenvironment, consequently triggering anti-cancer immune response.

\section{NAPSB highly expressed in hot tumors and may enhance immunotherapy response}

As mentioned earlier, solid tumors can be divided into hot tumors and cold tumors, and hot tumors respond to cancer immunotherapy $[14,51]$. Unsupervised clustering was conducted to classified HCC samples into hot tumors and cold tumors based on the hot tumor signature genes (Supplementary Table 14; Fig. 4a-d) [41]. Then, the expression of NAPSB was compared between hot and cold tumors and we found that it was overexpressed significantly in hot tumors (Fig. 4e), suggesting that NAPSB could play a role in distinct hot/cold tumor states and be associated with therapeutic response to immunotherapy. The same methods were used to validate above results in the ICGC cohort (Supplementary Fig. 3a-e).

In addition, NAPSB expression was found to be positively correlated with BTLA (B and T lymphocyte associated), CTLA-4 (cytotoxic T-lymphocyte-associated protein 4), IDO1 (indoleamine 2,3-dioxygenase 1), LAG-3 (lymphocyte activating 3), PD-1 (programmed cell death 1), PD-L1 (programmed cell death 1 ligand 1), TIGIT (T cell immunoreceptor with Ig and ITIM domains), and TIM-3 (T-cell immunoglobulin and mucin domain-containing protein 3) expression (Fig. 4f), which were well-known predictors of response to immunotherapy. Also, the enrichment scores of therapeutic signatures, predicting clinical response, were compared in NAPSB subgroups. As exhibited in Fig. 4g, i, NAPSB was negatively correlated with the enrichment scores of PPARG network, $\beta$-catenin signaling pathway, VEGFA and IDH1, which were all immunosuppressive gene signatures [15-17, 52]. However, in the NAPSB-high group, immunotherapypositive pathways such as IFN- $\gamma$-signature, APM-signal, EGFR-ligands, hypoxia and KDM6B (lysine demethylase 6b) were activated (Fig. 4h) [18-21, 53], indicating immune-activated state and beneficial to immunotherapy response. These observations were also validated using ICGC samples (Supplementary Fig. 3f-h).

The last but important, the role of the NAPSB in predicting the immune checkpoint blockade (ICB) response was explored in tow immunotherapy-related melanoma cohorts. In GSE91061, we found the ICB response rates were obviously higher in the NAPSB-high group than in the NAPSB-low group (Fig. 4j) and the expression of NAPSB was significantly high in response group (Fig. 4k). Similar results were observed in the GSE78220 cohort (Supplementary Fig. 3i). These evidences reconfirmed that NAPSB may be a valuable predictor of immunotherapy response across cancers. 


\section{NAPSB was associated with increased sensitivity to chemotherapy}

As for whether NAPSB plays a role in chemotherapy sensitivity, the correlation between NAPSB expression and IC50 of multiple drugs in cell lines was analyzed using data from GDSC and CTRP. Intriguingly, NAPSB expression was negatively associated with IC50 of most agents in GDSC and in CTRP (Fig. 5a and Supplementary Fig. 4; Supplementary Table 15), supporting that NAPSB can enhance the therapeutic response to chemotherapy. Two heat maps showed that the IC50 of some commonly used drugs is lower in the NAPSB-high group in GDSC and CTRP databases, respectively (Fig. 5b, c). Results above speculated that high expression of NAPSB is beneficial to the sensitive response of chemotherapy.

Thereafter, by analyzing GSE104580, a HCC cohort of transarterial chemoembolization (TACE), we found the expression of NAPSB was significantly higher in TACE response group (Fig. 5d) and the response rates were obviously higher in the NAPSB-high group than in the NAPSB-low group (Fig. 5e). This data further illustrated that high expression of NAPSB may be beneficial to chemotherapy response.

\section{Association of NAPSB with cell death of tumor cells}

Given that cell death had been reported in recent years to play a significant role in tumor therapy [54], we investigated the association between NAPSB and various forms of cell death, including pyroptosis, necroptosis, apoptosis, autophagy and ferroptosis, using ssGSEA. As showed in Fig. 6a-C, and E, NAPSB expression was markedly correlated with pyroptosis, apoptosis and necroptosis, but negatively correlated with ferroptosis. Autophagy had no correlation with NAPSB expression (Fig. 6d). We also verified these discoveries with ICGC cohort. In line with these findings, the correlations between NAPSB and several cell death forms were consistent with that in the ICGC cohort (Fig. 6f). In addition, the enrichment scores of pyroptosis, apoptosis and necroptosis in NAPSB-high groups were markedly higher than NAPSB-low groups (Fig. 6g). However, similar to results of TCGA, ferroptosis scores were lower in NAPSB-high group than in NAPSB-low group and autophagy scores had no significant differences in NAPSB subgroups. Among the above results, the correlation between NAPSB and pyroptosis was the most significant. Results above inferred that NAPSB may have a beneficial effect on immunotherapy and chemotherapy responses by promoting pyroptosis, necroptosis and apoptosis (PANoptosis) in tumor therapy.

\section{Discussion}

In this study, the potential biological functions of NAPSB has been comprehensively explored for the first time in HCC. We revealed NAPSB was downregulated and related to a better prognosis in HCC patients. Enrichment analyses suggested NAPSB was closely related to immune activation and further analysis demonstrated that NAPSB was associated with immunomodulators, higher T cell infiltration, hot tumor states, and corresponding better therapeutic efficacy. Mechanistically, we speculated that NAPSB might interact with PANoptosis thus improving the sensitivity of immunotherapy/chemotherapy in HCC. 
By analyzing the data from multiple public databases and Zhongnan cohort, our study obtained consistent results as previous research: NAPSB was downregulated in HCC [27]. Tan et al.'s study showed NAPSB was upregulated in PDAC and related to CD4 + T Cell infiltration [25]. Additionally, upregulation of NAPSB was also found in pre-eclampsia, a state of highly inflammatory activity [26]. It was expected that NAPSB overexpressed under inflammatory conditions. In line with these, enrichment analyses in this study showed the genes most related to NAPSB were enriched in immune cell receptor signaling pathway and inflammatory response in our study.

A more important part of this study was to comprehensively clarify the immunological role of NAPSB in HCC immune microenvironment. MHC molecules represent antigen presentation and processing capacity, while chemokines and receptors recruit effector TIICs such as CD8 + T cells, TH17 cells, and antigenpresenting cells $[55,56]$, which may upregulate the activities of the cancer-immunity cycle subsequently [57]. In our study, NAPSB was found to be positively correlated with these immunomodulators, suggesting that NAPSB promotes immune activation, which is consistent with the results of enrichment analyses above. In addition, NAPSB expression had a positive correlation with the abundance of infiltrating CD8 + T cells, CD $4+T$ cells, B cells, NK cells, macrophages, and DCs in HCC, verified by different algorithms and external validation cohort. Currently, the prognosis of HCC is known to be related to the infiltration and activation of immune cells[58, 59], whose presence participate in a hot (inflamed) TME [60, 61], supporting the observation that NAPSB can stimulate the immune response in the TME and play an antitumor role in HCC, thereby prolonging survival. This could also be used to explain the results of this study: high expression of NAPSB was associated with better prognosis of HCC. Additionally, we observed NAPSB was positively related to the $T$ cell inflamed score, as well as several critical steps of the cancer immunity cycle. Since both TIS and cancer immunity cycle reflect the T cell infiltration and anti-cancer immune response of our body [38, 40], these results reaffirmed and extended the close relationship between NAPSB and an immune-hot and inflamed TME.

T-cell infiltration, molecular characteristics of immune activation, and anti-tumor response are characteristics of hot tumors [51, 62], so we speculated NAPSB can play a role in distinct hot/cold tumor states based on the above results. Here, NAPSB was highly expressed in hot tumor samples consistently. Not only that, NAPSB was significantly positively correlated with ICB therapeutic targets, such as PD-L1, PD-1, and CTLA-4. Better clinical response to ICB is another character of hot tumors due to more active immune molecules [63]. Together, NAPSB could distinguish between hot and cold tumors and facilitate immunotherapeutic responses. Meanwhile, we discovered that NAPSB high group was activated in immune-activated pathways, such as the IFN- $\gamma$ signature, APM signal, EGFR ligands, and hypoxia [1821]. These pathways had been revealed to stimulate the infiltration of TIICs by increasing the expression of immunomodulators, contributing to an inflamed TME and resulting in better clinical responses to immunotherapy. On the contrary, NAPSB was opposite to several reported immunosuppressive oncogenic pathways, such as the $\beta$-catenin, PPARG, and IDH1 pathways [15-17]. These outcomes not only demonstrated that NAPSB can improve the immunotherapy response, but also reconfirmed the role of NAPSB in activation of immune activity as discussed above. 
The main treatments for advanced HCC are still chemotherapy and targeted drugs, among which first-line drugs include doxorubicin, fluorouracil, and sorafenib, etc [2], improving the five-year survival rates of HCC patients [64]. TACE is a treatment for liver cancer often applying doxorubicin or cisplatin as intra-arterial injection of cytotoxic agents [65]. In our study, we proved that NAPSB was negatively correlated with IC50 of a variety of drugs in GDSC and CTRP databases, but overexpressed in TACE responders as expected, strongly inferring high NAPSB expression can improve the sensitivity of chemotherapy. NAPSB may be utilized as a promising predictive marker for chemotherapy as drug resistance is common at present [ 9 , 66]. Recently, studies have focused on the interactions between tumor cell death and sensitivity or resistance of anticancer therapy. For instance, Makin et al. created that apoptosis was considered as the predominant form of regulated cell death responsible for tumor therapies [67]. And Carina et al.'s study revealed sorafenib therapy induced pyroptosis in $\mathrm{M \Phi}$ and thereby unleashing an NK-cell response against HCC tumors [68]. Instead, autophagy, this cell death form participates in the progression of HCC and the resistance of HCC cells to sorafenib [49, 69]. In our study, we revealed NAPSB was positively correlated with pyroptosis, apoptosis and necroptosis (PANoptosis), but had no correlation with autophagy, suggesting that NAPSB may promote cell death synergistically with drugs to improve the sensitivity of chemotherapy.

Despite these findings, there are existing limitations. First, the study was primarily carried out using bioinformatics methods; further experiments are needed to support these findings. To remedy this deficiency, the main conclusions of this study were confirmed by several methods and external validation. For instance, the differential expression of NAPSB was compared across multiple cohorts and the association of NAPSB with immune infiltration was demonstrated by sSGSEA and six other independent algorithms. Second, although this study showed NAPSB could serve as an independent predictor of disease-free and progression-free survival outcomes of HCC patients from the TCGA cohort, we did not validate this in another cohort due to a lack of such datasets with complete clinical information. Third, further in-depth studies are required to explore the interactions of NAPSB, PANoptosis and chemotherapy.

In conclusion, our study is the first comprehensive analysis to demonstrate that NAPSB expression shaped an immuno-hot and inflamed TME in HCC; NAPSB could be considered a predictor of disease-free and progression-free survival outcomes in patients with HCC; NAPSB can also predict the clinical response to ICB and chemotherapy. These findings will provide important insights for the development of cytokine-based therapy for cancer treatment.

\section{Abbreviations}




\begin{tabular}{ll} 
APM & Antigen processing and presenting machinery \\
\hline BLCA & Bladder urothelial carcinoma \\
\hline BRCA & Breast invasive carcinoma \\
\hline BTLA & B and T lymphocyte associated \\
\hline COAD & Colon adenocarcinoma \\
\hline CTLA4 & Cytotoxic T-lymphocyte-associated protein 4 \\
\hline CTRP & Cancer therapeutics response portal \\
\hline DCs & Dendritic cells \\
\hline DEGs & Differentially expressed genes \\
\hline DFI & Disease-free interval \\
\hline EGFR & Epidermal growth factor receptor \\
\hline ESCA & Esophageal carcinoma \\
\hline GDSC & Genomics of drug sensitivity in cancer \\
\hline GEO & Gene expression omnibus \\
\hline GO & Gene ontology \\
\hline GSEA & Gene set enrichment analysis \\
\hline GSVA & Gene set variation analysis \\
\hline HCC & Hepatocellular carcinoma \\
\hline IC50 & Half maximal inhibitory concentration \\
\hline ICGC & International cancer genome consortium \\
\hline ICls & Immune checkpoint inhibitors \\
\hline IDH1 & Isocitrate dehydrogenase 1 \\
\hline IDO1 & Indoleamine 2,3-dioxygenase 1 \\
\hline IFN-Y & interferon-gamma \\
\hline KDM6B & Lysine demethylase 6b \\
\hline KEGG & Kyoto encyclopedia of genes and genomes \\
\hline KIRP & Kidney chromosome \\
\hline KIillary cell carcinoma \\
\hline K
\end{tabular}




\begin{tabular}{ll}
\hline LAG-3 & Lymphocyte activating 3 \\
\hline LIHC & Liver hepatocellular carcinoma \\
\hline LncRNAs & Long non-coding RNAs \\
\hline LUAD & Lung adenocarcinoma \\
\hline LUSC & Lung squamous cell carcinoma \\
\hline MHC & Major histocompatibility complex \\
\hline NAPSB & Napsin b aspartic peptidase, pseudogene \\
\hline OS & Overall survival \\
\hline PANoptosis & Pyroptosis, apoptosis and necrosis \\
\hline PDAC & Pancreatic ductal adenocarcinoma \\
\hline PD-1 & Programmed cell death 1 \\
\hline PD-L1 & Programmed cell death 1 ligand 1 \\
\hline PFI & Progression-free interval \\
\hline PPARG & Peroxisome proliferator activated receptor gamma \\
\hline qRT-PCR & Quantitative reverse transcription polymerase chain reaction \\
\hline READ & Rectal adenocarcinoma \\
\hline TACE & Transcatheter arterial chemoembolization \\
\hline TIGIT & T cell immunoreceptor with Ig and ITIM domains \\
\hline TIICs & Tumor-infiltrating immune cells \\
\hline TIM3 & T-cell immunoglobulin and mucin domain-containing protein 3 \\
\hline TIS & T-cell inflammation score \\
\hline TCGA & The cancer genome atlas \\
\hline TME & Tumor microenvironment \\
\hline VEGFA & Vascular endothelial growth factor a \\
\hline
\end{tabular}

\section{Declarations}

\section{Acknowledgements}

We thank Hai-Hang Nie and the colleagues from the department of Gastroenterology of Zhongnan Hospital for their generous help in this research design. 


\section{Author Contributions}

Q.Z. H.W. and J.F. designed the study. X.L., K.L. and F.W. collected the data. Y.N. and K.L. analyzed the data. Y.N., H.W. and K.L prepared the figures. X.L. and F.W. prepared the tables. X.J., Z.Z. and L.L. collected the human specimens. Y.D., K.L. and Y.X. carried out the experiments. H.W. Q.Z. and J.F. supervised the data and provided statistical advice. Y.N., K.L. and X.L. wrote the article. F.W. and H.W. reviewed the article. All authors read and approved the final manuscript.

\section{Funding}

This work was supported by the following projects: The National Natural Science Foundation of China (grant no.81800505, 81870390). Engineering construction project of improving diagnosis and treatment ability of difficult diseases (oncology) (grant no. ZLYNXM202017).

\section{Data Availability}

Publicly available datasets were analyzed in this study. These data can be found here: https://portal.gdc.cancer.gov/, https://icgc.org/, and https://www.ncbi.nlm.nih.gov/geo/. The supplementary material for this article can be found online. All processed data and R codes used in this study can be obtained from the corresponding author on reasonable request.

\section{Conflict of interests}

The authors declare that they have no competing interests.

\section{Ethical approval}

The study was approved by the Research Ethics Committee of Zhongnan Hospital of Wuhan University, and the written informed consent was obtained from all patients.

\section{References}

1. Sung H, Ferlay J, Siegel RL, et al. (2021) Global Cancer Statistics 2020: GLOBOCAN Estimates of Incidence and Mortality Worldwide for 36 Cancers in 185 Countries. CA Cancer J Clin 713:209-249. https://doi.org/10.3322/caac.21660.

2. Gordan JD, Kennedy EB, Abou-Alfa GK, et al. (2020) Systemic Therapy for Advanced Hepatocellular Carcinoma: ASCO Guideline. J Clin Oncol 3836:4317-4345.https://doi.org/10.1200/JC0.20.02672.

3. Kudo M (2019) Scientific Rationale for Combination Immunotherapy of Hepatocellular Carcinoma with Anti-PD-1/PD-L1 and Anti-CTLA-4 Antibodies. Liver cancer 86:413- 
426.https://doi.org/10.1159/000503254.

4. Robert C, Schachter J, Long GV, et al. (2015) Pembrolizumab versus Ipilimumab in Advanced Melanoma. The New England journal of medicine 37226:2521-

2532.https://doi.org/10.1056/NEJMoa1503093.

5. Nayman AH, Siginc H, Zemheri E, et al. (2019) Dual-Inhibition of mTOR and Bcl-2 Enhances the Antitumor Effect of Everolimus against Renal Cell Carcinoma In Vitro and In Vivo. Journal of Cancer 106:1466-1478.https://doi.org/10.7150/jca.29192.

6. Rosenberg JE, Hoffman-Censits J, Powles T, et al. (2016) Atezolizumab in patients with locally advanced and metastatic urothelial carcinoma who have progressed following treatment with platinum-based chemotherapy: a single-arm, multicentre, phase 2 trial. Lancet (London, England) 38710031:1909-1920.https://doi.org/10.1016/s0140-6736(16)00561-4.

7. O'Donnell JS, Long GV, Scolyer RA, Teng MW, \& Smyth MJ (2017) Resistance to PD1/PDL1 checkpoint inhibition. Cancer treatment reviews 52:71-81.https://doi.org/10.1016/j.ctrv.2016.11.007.

8. Mariathasan S, Turley SJ, Nickles D, et al. (2018) TGF $\beta$ attenuates tumour response to PD-L1 blockade by contributing to exclusion of T cells. Nature 5547693:544548.https://doi.org/10.1038/nature25501.

9. Chen S, Cao Q, Wen W, \& Wang H (2019) Targeted therapy for hepatocellular carcinoma: Challenges and opportunities. Cancer letters 460:1-9.https://doi.org/10.1016/j.canlet.2019.114428.

10. Xiao Y \& Yu D (2021) Tumor microenvironment as a therapeutic target in cancer. Pharmacology \& therapeutics 221:107753.https://doi.org/10.1016/j.pharmthera.2020.107753.

11. Lambrechts $D$, Wauters $E$, Boeckx B, et al. (2018) Phenotype molding of stromal cells in the lung tumor microenvironment. Nature medicine 248:1277-1289.https://doi.org/10.1038/s41591-0180096-5.

12. Chen DS \& Mellman I (2017) Elements of cancer immunity and the cancer-immune set point. Nature 5417637:321-330.https://doi.org/10.1038/nature21349.

13. Ji RR, Chasalow SD, Wang L, et al. (2012) An immune-active tumor microenvironment favors clinical response to ipilimumab. Cancer immunology, immunotherapy : Cll 617:10191031.https://doi.org/10.1007/s00262-011-1172-6.

14. Too NSH, Ho NCW, Adine C, lyer NG, \& Fong ELS (2021) Hot or cold: Bioengineering immune contextures into in vitro patient-derived tumor models. Advanced drug delivery reviews 175:113791.https://doi.org/10.1016/j.addr.2021.05.001.

15. Ruiz de Galarreta M, Bresnahan E, Molina-Sánchez P, et al. (2019) $\beta$-Catenin Activation Promotes Immune Escape and Resistance to Anti-PD-1 Therapy in Hepatocellular Carcinoma. Cancer discovery 98:1124-1141.https://doi.org/10.1158/2159-8290.Cd-19-0074.

16. To JC, Chiu AP, Tschida BR, et al. (2021) ZBTB20 regulates WNT/CTNNB1 signalling pathway by suppressing PPARG during hepatocellular carcinoma tumourigenesis. JHEP reports : innovation in hepatology 32:100223.https://doi.org/10.1016/j.jhepr.2020.100223. 
17. Saha SK, Parachoniak CA, Ghanta KS, et al. (2014) Mutant IDH inhibits HNF-4a to block hepatocyte differentiation and promote biliary cancer. Nature 5137516:110-

114.https://doi.org/10.1038/nature13441.

18. Riaz N, Havel JJ, Makarov V, et al. (2017) Tumor and Microenvironment Evolution during Immunotherapy with Nivolumab. Cell 1714:934-949.e916.https://doi.org/10.1016/j.cell.2017.09.028.

19. Wang S, He Z, Wang X, Li H, \& Liu XS (2019) Antigen presentation and tumor immunogenicity in cancer immunotherapy response prediction. eLife 8.https://doi.org/10.7554/eLife.49020.

20. Qiao M, Jiang T, Liu X, et al. (2021) Immune Checkpoint Inhibitors in EGFR-Mutated NSCLC: Dusk or Dawn? Journal of thoracic oncology : official publication of the International Association for the Study of Lung Cancer 168:1267-1288.https://doi.org/10.1016/j.jtho.2021.04.003.

21. Taylor CT \& Colgan SP (2017) Regulation of immunity and inflammation by hypoxia in immunological niches. Nature reviews. Immunology 1712:774-

785.https://doi.org/10.1038/nri.2017.103.

22. Proudfoot N (1980) Pseudogenes. Nature 2865776:840-841.https://doi.org/10.1038/286840a0.

23. Yue $\mathrm{C}$, Ren $\mathrm{Y}, \mathrm{Ge} \mathrm{H}$, et al. (2019) Comprehensive analysis of potential prognostic genes for the construction of a competing endogenous RNA regulatory network in hepatocellular carcinoma. OncoTargets and therapy 12:561-576.https://doi.org/10.2147/ott.S188913.

24. Bao L, Li P, Zhao H, et al. (2021) Pseudogene PLGLA exerts anti-tumor effects on hepatocellular carcinoma through modulating miR-324-3p/GLYATL1 axis. Digestive and liver disease : official journal of the Italian Society of Gastroenterology and the Italian Association for the Study of the Liver.https://doi.org/10.1016/j.dld.2021.10.003.

25. Tan Z, Lei Y, Zhang B, et al. (2021) Analysis of Immune-Related Signatures Related to CD4+ T Cell Infiltration With Gene Co-Expression Network in Pancreatic Adenocarcinoma. Frontiers in oncology 11:674897.https://doi.org/10.3389/fonc.2021.674897.

26. He J, Liu K, Hou X, \& Lu J (2021) Identification and validation of key non-coding RNAs and mRNAs using co-expression network analysis in pre-eclampsia. Medicine 10014:e25294.https://doi.org/10.1097/md.0000000000025294.

27. Dai M, Chen $S$, Wei $X$, et al. (2017) Diagnosis, prognosis and bioinformatics analysis of IncRNAs in hepatocellular carcinoma. Oncotarget 856:95799-95809.https://doi.org/10.18632/oncotarget.21329.

28. Charoentong P, Finotello F, Angelova M, et al. (2017) Pan-cancer Immunogenomic Analyses Reveal Genotype-Immunophenotype Relationships and Predictors of Response to Checkpoint Blockade. Cell reports 181:248-262.https://doi.org/10.1016/j.celrep.2016.12.019.

29. Yoshihara K, Shahmoradgoli M, Martínez E, et al. (2013) Inferring tumour purity and stromal and immune cell admixture from expression data. Nature communications 4:2612.https://doi.org/10.1038/ncomms3612.

30. Li T, Fu J, Zeng Z, et al. (2020) TIMER2.0 for analysis of tumor-infiltrating immune cells. Nucleic acids research 48W1:W509-w514.https://doi.org/10.1093/nar/gkaa407. 
31. Chen B, Khodadoust MS, Liu CL, Newman AM, \& Alizadeh AA (2018) Profiling Tumor Infiltrating Immune Cells with CIBERSORT. Methods in molecular biology (Clifton, N.J.) 1711:243259.https://doi.org/10.1007/978-1-4939-7493-1_12.

32. Finotello F, Mayer C, Plattner C, et al. (2019) Molecular and pharmacological modulators of the tumor immune contexture revealed by deconvolution of RNA-seq data. Genome medicine 111:34.https://doi.org/10.1186/s13073-019-0638-6.

33. Racle J, de Jonge K, Baumgaertner P, Speiser DE, \& Gfeller D (2017) Simultaneous enumeration of cancer and immune cell types from bulk tumor gene expression data. eLife 6.https://doi.org/10.7554/eLife.26476.

34. Aran D, Hu Z, \& Butte AJ (2017) xCell: digitally portraying the tissue cellular heterogeneity landscape. Genome biology 181:220.https://doi.org/10.1186/s13059-017-1349-1.

35. Becht E, Giraldo NA, Lacroix L, et al. (2016) Estimating the population abundance of tissue-infiltrating immune and stromal cell populations using gene expression. Genome biology

171:218.https://doi.org/10.1186/s13059-016-1070-5.

36. Hu J, Yu A, Othmane B, et al. (2021) Siglec15 shapes a non-inflamed tumor microenvironment and predicts the molecular subtype in bladder cancer. Theranostics 117:3089-

3108.https://doi.org/10.7150/thno.53649.

37. Brummelman J, Pilipow K, \& Lugli E (2018) The Single-Cell Phenotypic Identity of Human CD8(+) and CD4(+) T Cells. International review of cell and molecular biology 341:63124.https://doi.org/10.1016/bs.ircmb.2018.05.007.

38. Chen DS \& Mellman I (2013) Oncology meets immunology: the cancer-immunity cycle. Immunity 391:1-10.https://doi.org/10.1016/j.immuni.2013.07.012.

39. Xu L, Deng C, Pang B, et al. (2018) TIP: A Web Server for Resolving Tumor Immunophenotype Profiling. Cancer research 7823:6575-6580.https://doi.org/10.1158/0008-5472.Can-18-0689.

40. Ayers M, Lunceford J, Nebozhyn M, et al. (2017) IFN-y-related mRNA profile predicts clinical response to PD-1 blockade. The Journal of clinical investigation 1278:29302940.https://doi.org/10.1172/jci91190.

41. Dong C, Dang D, Zhao X, et al. (2021) Integrative Characterization of the Role of IL27 In Melanoma Using Bioinformatics Analysis. Frontiers in immunology 12:713001.https://doi.org/10.3389/fimmu.2021.713001.

42. Wilkerson MD \& Hayes DN (2010) ConsensusClusterPlus: a class discovery tool with confidence assessments and item tracking. Bioinformatics (Oxford, England) 2612:15721573.https://doi.org/10.1093/bioinformatics/btq170.

43. Hänzelmann S, Castelo R, \& Guinney J (2013) GSVA: gene set variation analysis for microarray and RNA-seq data. BMC bioinformatics 14:7.https://doi.org/10.1186/1471-2105-14-7.

44. Yang W, Soares J, Greninger P, et al. (2013) Genomics of Drug Sensitivity in Cancer (GDSC): a resource for therapeutic biomarker discovery in cancer cells. Nucleic acids research 41Database issue:D955-961.https://doi.org/10.1093/nar/gks1111. 
45. Seashore-Ludlow B, Rees MG, Cheah JH, et al. (2015) Harnessing Connectivity in a Large-Scale Small-Molecule Sensitivity Dataset. Cancer discovery 511:1210-1223.https://doi.org/10.1158/21598290.Cd-15-0235.

46. Ye Y, Dai Q, \& Qi H (2021) A novel defined pyroptosis-related gene signature for predicting the prognosis of ovarian cancer. Cell death discovery 71:71.https://doi.org/10.1038/s41420-021-00451$\mathrm{x}$.

47. Yu GP, Xiao QY, Shi ZQ, et al. (2015) Genetic polymorphisms in apoptosis-related genes and the prognosis of hepatocellular carcinoma. American journal of cancer research 510:3249-3259.

48. Wang N \& Liu D (2021) Identification and Validation a Necroptosis-related Prognostic Signature and Associated Regulatory Axis in Stomach Adenocarcinoma. OncoTargets and therapy 14:53735383.https://doi.org/10.2147/ott.S342613.

49. Cui J, Shen HM, \& Lim LHK (2020) The Role of Autophagy in Liver Cancer: Crosstalk in Signaling Pathways and Potential Therapeutic Targets. Pharmaceuticals (Basel, Switzerland) 1312.https://doi.org/10.3390/ph13120432.

50. Liang JY, Wang DS, Lin HC, et al. (2020) A Novel Ferroptosis-related Gene Signature for Overall Survival Prediction in Patients with Hepatocellular Carcinoma. International journal of biological sciences 1613:2430-2441.https://doi.org/10.7150/ijbs.45050.

51. Duan Q, Zhang H, Zheng J, \& Zhang L (2020) Turning Cold into Hot: Firing up the Tumor Microenvironment. Trends in cancer 67:605-618.https://doi.org/10.1016/j.trecan.2020.02.022.

52. Claesson-Welsh L \& Welsh M (2013) VEGFA and tumour angiogenesis. Journal of internal medicine 2732:114-127.https://doi.org/10.1111/joim.12019.

53. Xu T, Schutte A, Jimenez L, et al. (2021) Kdm6b Regulates the Generation of Effector CD8(+) T Cells by Inducing Chromatin Accessibility in Effector-Associated Genes. Journal of immunology (Baltimore, Md. : 1950) 2069:2170-2183.https://doi.org/10.4049/jimmunol.2001459.

54. Tang R, Xu J, Zhang B, et al. (2020) Ferroptosis, necroptosis, and pyroptosis in anticancer immunity. Journal of hematology \& oncology 131:110.https://doi.org/10.1186/s13045-020-00946-7.

55. Ulfig A, Bader V, Varatnitskaya M, et al. (2021) Hypochlorous acid-modified human serum albumin suppresses MHC class II - dependent antigen presentation in pro-inflammatory macrophages. Redox biology 43:101981.https://doi.org/10.1016/j.redox.2021.101981.

56. Nagarsheth N, Wicha MS, \& Zou W (2017) Chemokines in the cancer microenvironment and their relevance in cancer immunotherapy. Nature reviews. Immunology 179:559572.https://doi.org/10.1038/nri.2017.49.

57. Seya T, Shime H, Takeda Y, et al. (2015) Adjuvant for vaccine immunotherapy of cancer--focusing on Toll-like receptor 2 and 3 agonists for safely enhancing antitumor immunity. Cancer science 10612:1659-1668.https://doi.org/10.1111/cas.12824.

58. Wu C, Lin J, Weng Y, et al. (2020) Myeloid signature reveals immune contexture and predicts the prognosis of hepatocellular carcinoma. The Journal of clinical investigation 1309:46794693.https://doi.org/10.1172/jci135048. 
59. Liu T, Tan J, Wu M, et al. (2021) High-affinity neoantigens correlate with better prognosis and trigger potent antihepatocellular carcinoma (HCC) activity by activating CD39(+)CD8(+) T cells. Gut 7010:1965-1977.https://doi.org/10.1136/gutjnl-2020-322196.

60. Bindea G, Mlecnik B, Tosolini M, et al. (2013) Spatiotemporal dynamics of intratumoral immune cells reveal the immune landscape in human cancer. Immunity 394:782-

795.https://doi.org/10.1016/j.immuni.2013.10.003.

61. van der Woude LL, Gorris MAJ, Halilovic A, Figdor CG, \& de Vries IJM (2017) Migrating into the Tumor: a Roadmap for T Cells. Trends in cancer 311:797808.https://doi.org/10.1016/j.trecan.2017.09.006.

62. Gajewski TF (2015) The Next Hurdle in Cancer Immunotherapy: Overcoming the Non-T-Cell-Inflamed Tumor Microenvironment. Seminars in oncology 424:663671.https://doi.org/10.1053/j.seminoncol.2015.05.011.

63. Pelly VS, Moeini A, Roelofsen LM, et al. (2021) Anti-Inflammatory Drugs Remodel the Tumor Immune Environment to Enhance Immune Checkpoint Blockade Efficacy. Cancer discovery 1110:26022619.https://doi.org/10.1158/2159-8290.Cd-20-1815.

64. Llovet JM, Montal R, Sia D, \& Finn RS (2018) Molecular therapies and precision medicine for hepatocellular carcinoma. Nature reviews. Clinical oncology 1510:599616.https://doi.org/10.1038/s41571-018-0073-4.

65. Chang Y, Jeong SW, Young Jang J, \& Jae Kim Y (2020) Recent Updates of Transarterial Chemoembolilzation in Hepatocellular Carcinoma. International journal of molecular sciences 2121.https://doi.org/10.3390/ijms21218165.

66. Johnson PJ, Qin S, Park JW, et al. (2013) Brivanib versus sorafenib as first-line therapy in patients with unresectable, advanced hepatocellular carcinoma: results from the randomized phase III BRISKFL study. J Clin Oncol 3128:3517-3524.https://doi.org/10.1200/jco.2012.48.4410.

67. Makin G \& Dive C (2001) Apoptosis and cancer chemotherapy. Trends in cell biology 1111:S2226.https://doi.org/10.1016/s0962-8924(01)02124-9.

68. Hage C, Hoves S, Strauss L, et al. (2019) Sorafenib Induces Pyroptosis in Macrophages and Triggers Natural Killer Cell-Mediated Cytotoxicity Against Hepatocellular Carcinoma. Hepatology (Baltimore, Md.) 704:1280-1297.https://doi.org/10.1002/hep.30666.

69. Xu WP, Liu JP, Feng JF, et al. (2020) miR-541 potentiates the response of human hepatocellular carcinoma to sorafenib treatment by inhibiting autophagy. Gut 697:13091321.https://doi.org/10.1136/gutjnl-2019-318830.

\section{Figures}




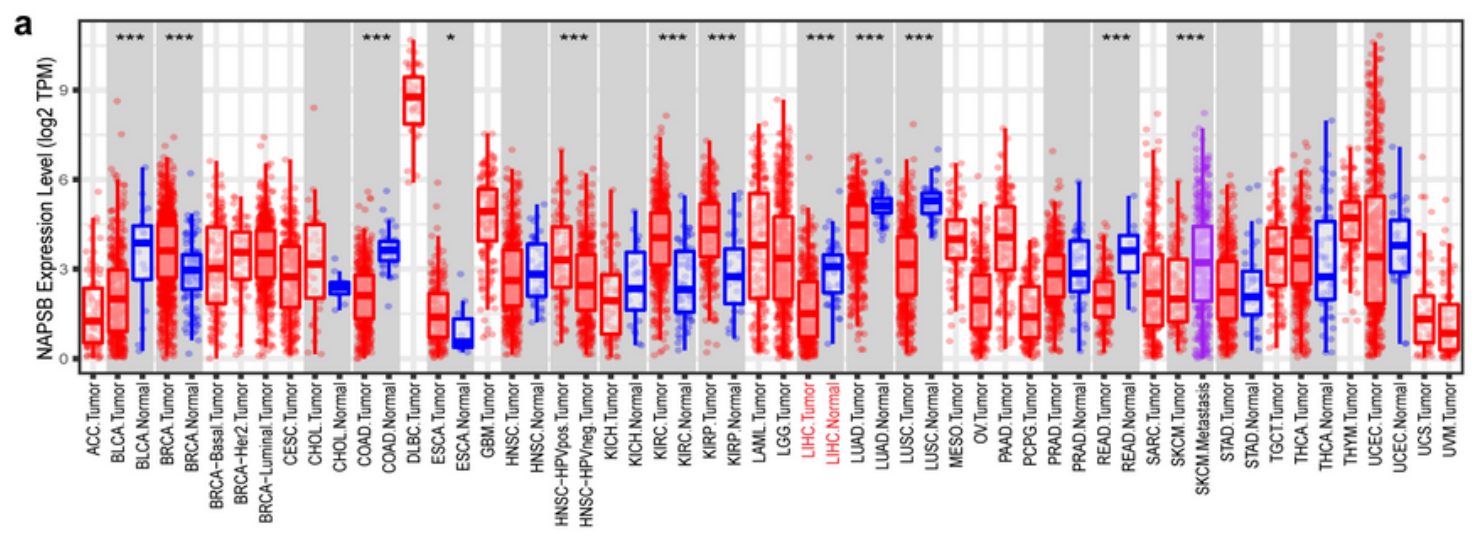

b

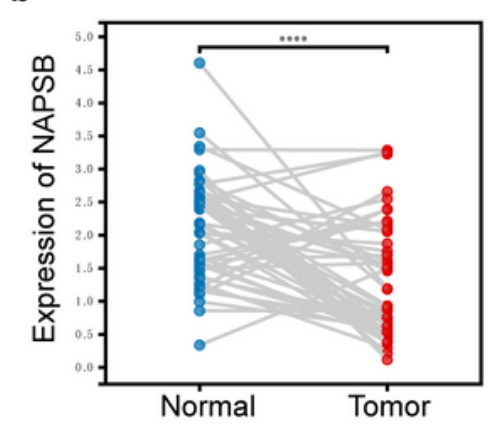

d

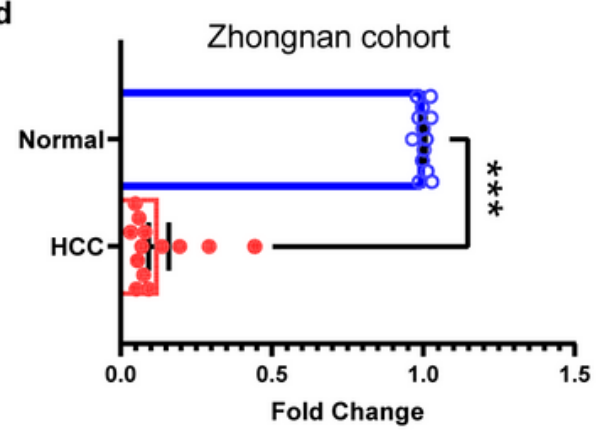

c

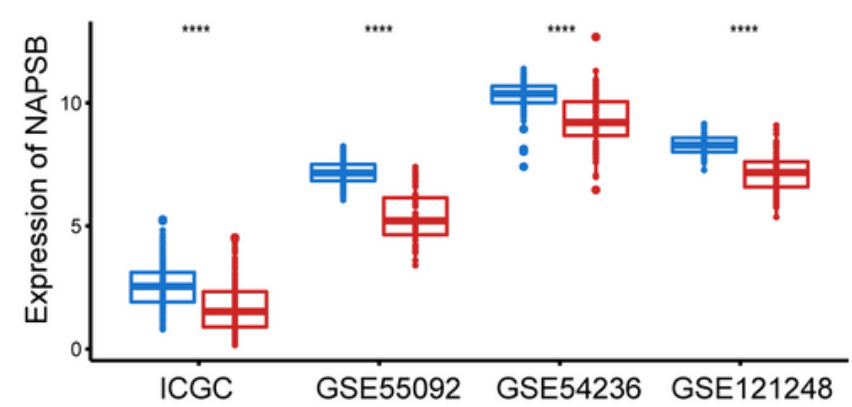

f

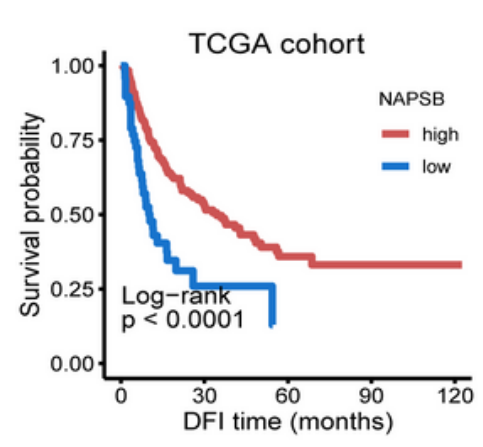

g

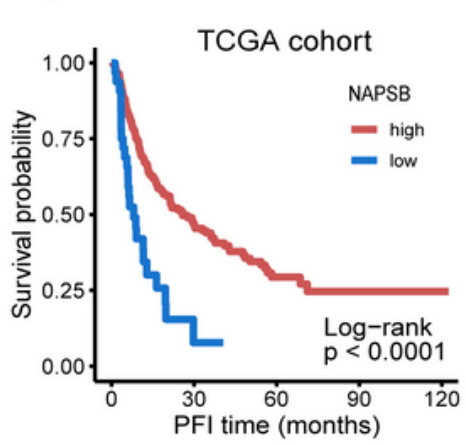

TCGA cohort

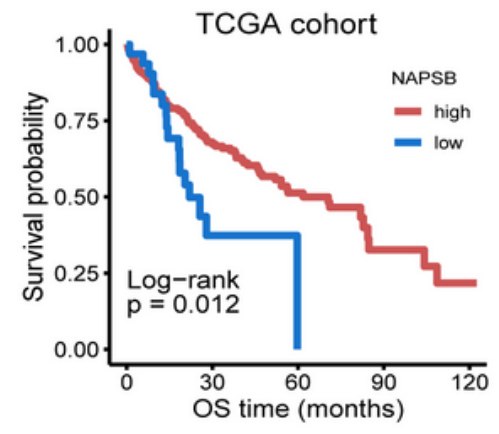

h

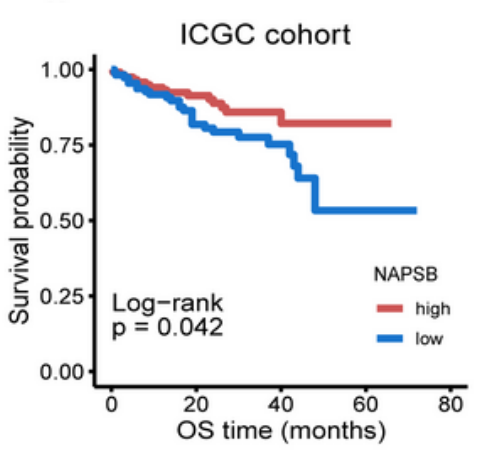

\section{Figure 1}

Differential expression and prognosis value of NAPSB in pan-cancer and liver hepatocellular carcinoma (LIHC). a NAPSB expression levels in different tumor types were measured using TIMER. b Paired Student's t-test analysis of NAPSB expression in TCGA paired samples. c NAPSB expression was significantly higher in normal tissues than in hepatocellular carcinoma in ICGC, GSE55092, GSE54236, and GSE121248 cohorts. d In Zhongnan cohort, lower NAPSB expression was seen in hepatocellular 
carcinoma compared with adjacent normal tissues $(\mathrm{N}=13)$. The expression of NAPSB was compared with a standard reference control and relative quantities $(\mathrm{RQ})$ were calculated based on the $\Delta \Delta \mathrm{Ct}$ method. e-h Kaplan-Meier analysis of NAPSB expression based on overall survival (OS), disease-free interval (DFI), progression-free interval (PFI) in TCGA cohort and overall survival (OS) in ICGC cohort. ns, no

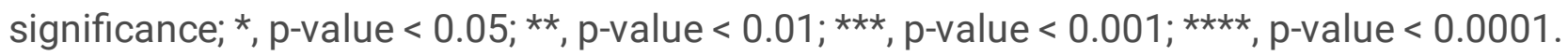

a

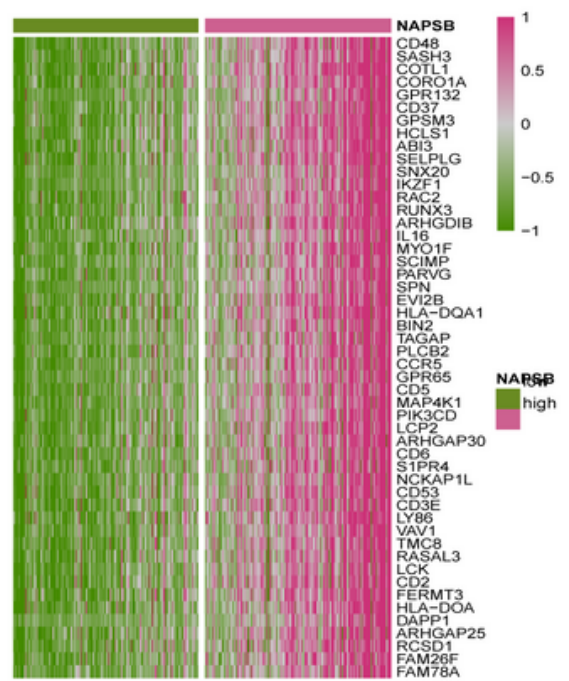

d

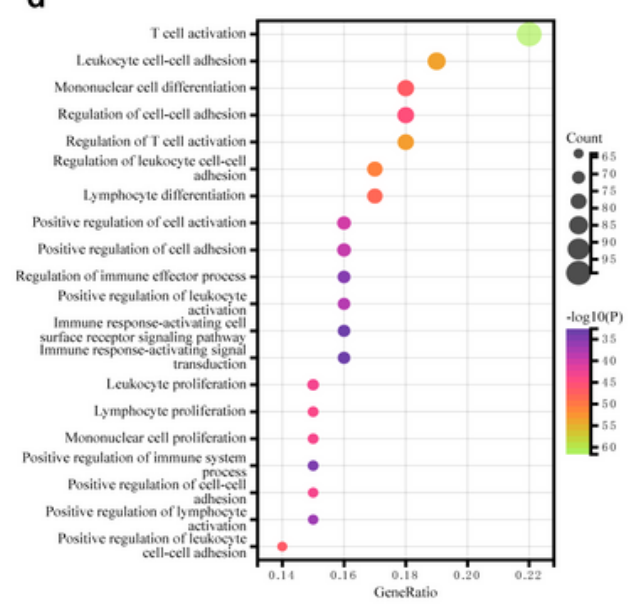

b

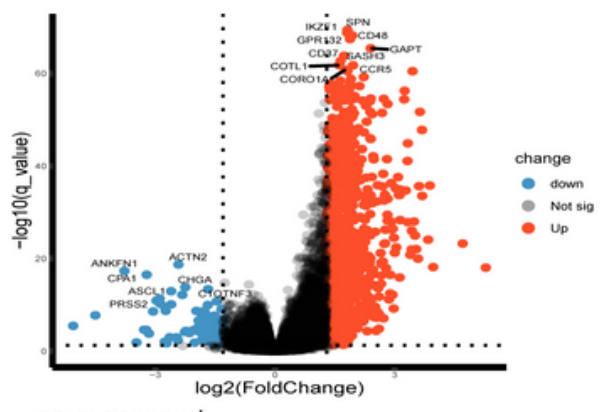

c

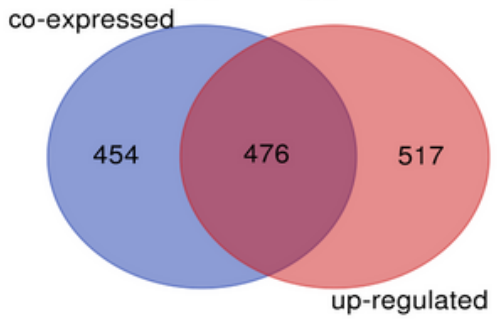

e

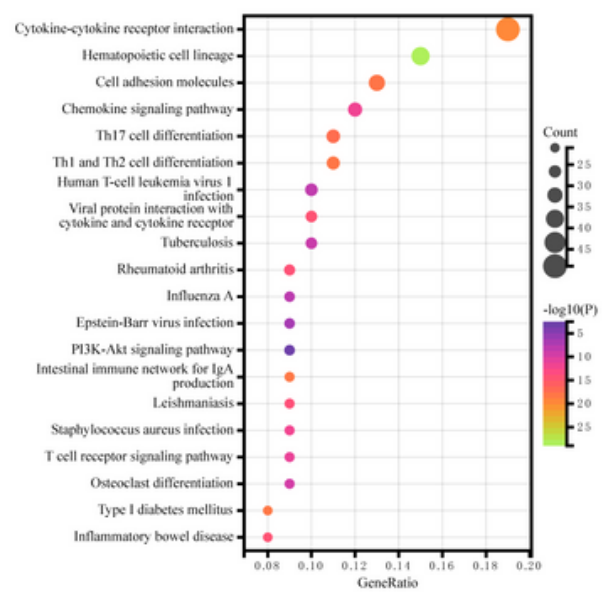

f
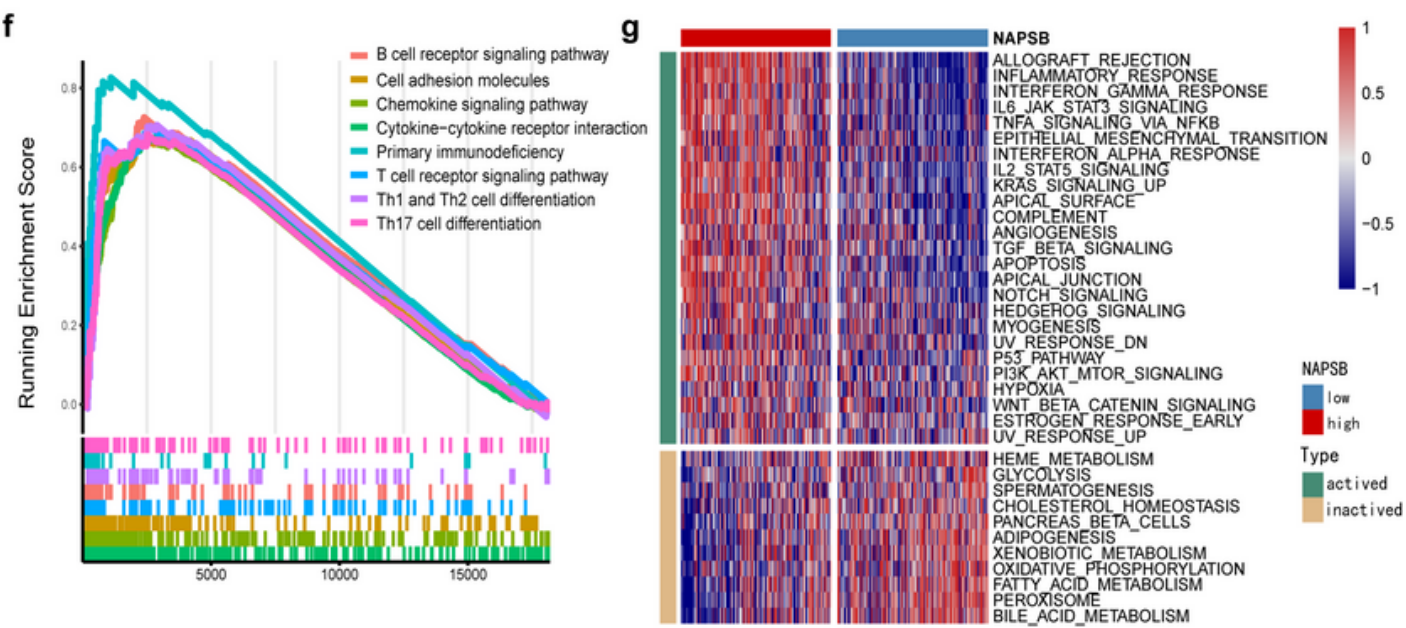

Figure 2 
Enrichment analysis of NAPSB biological function in hepatocellular carcinoma (HCC). a The heat map shows the top 50 genes positively related to NAPSB in HCC. $\mathbf{b}$ Volcano plot of DEGs between NAPSB-high and NAPSB-low HCC tissues. c Venn diagram of co-expressed genes and upregulated DEGs. $\mathbf{d}$ The top 20 gene ontology (GO) terms for the most closely related genes to NAPSB. e The top 20 Kyoto Encyclopedia of Genes and Genomes (KEGG) terms for the most closely related genes to NAPSB. $\mathbf{f}$ Gene Set Enrichment Analysis (GSEA) shows significant signaling pathways between NAPSB-high and NAPSB-low HCC tissues (The gene sets of "c5.cp.kegg.v7.4.symbols"). $\mathbf{g}$ Gene Set Variation Analysis (GSVA) between NAPSB-high and NAPSB-low HCC tissues (The gene sets of "h.all.v7.4.symbols"). 


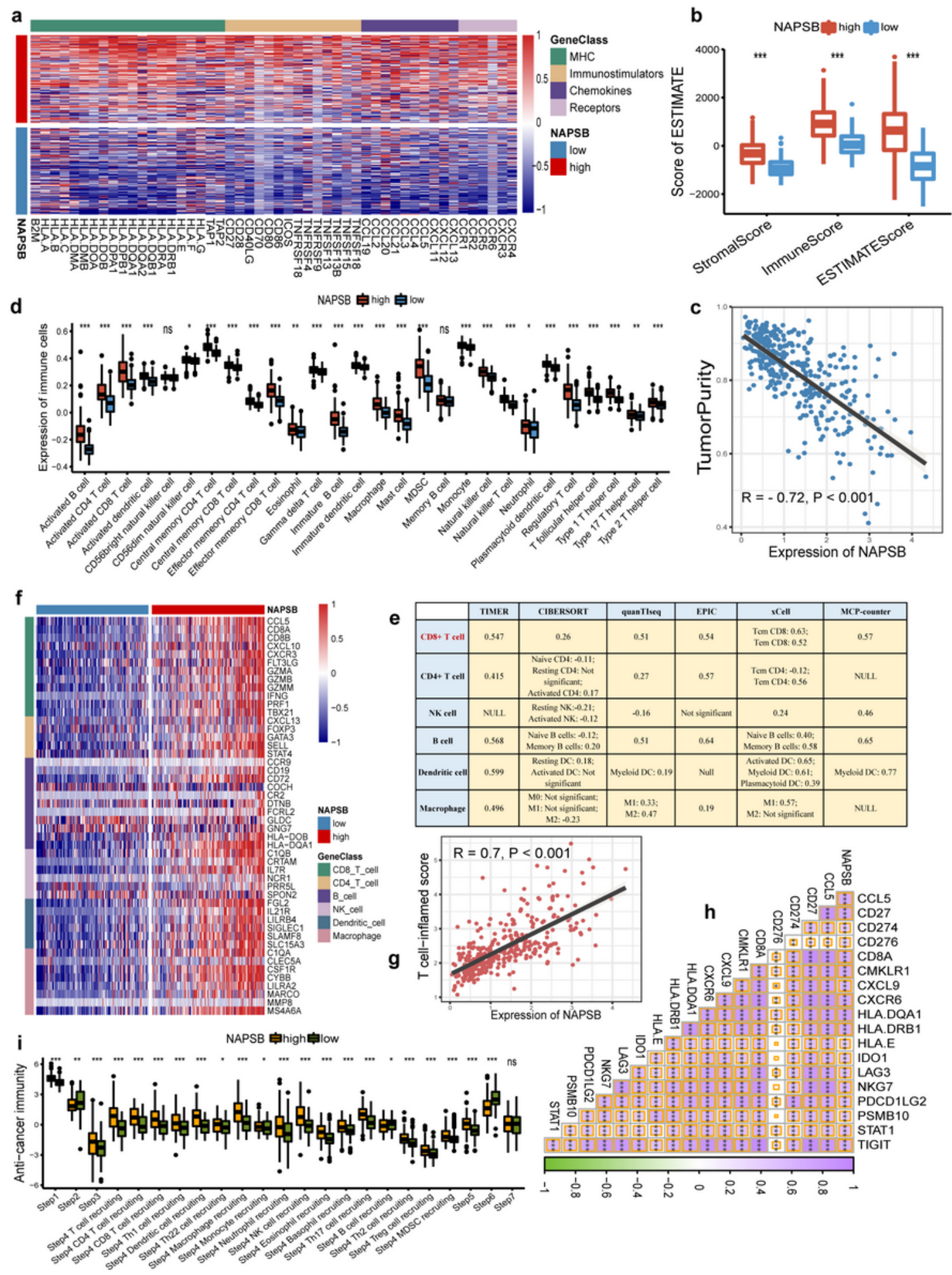

Figure 3

NAPSB shapes an inflamed TME in HCC. a Expression levels of 50 immunomodulators (MHC, immunostimulators, chemokines, and receptors) in the NAPSB-high and NAPSB-low groups in HCC. $\mathbf{b}$ Distribution of stromal score, immune score, and ESTIMATE score calculated using the ESTIMATE algorithm in the NAPSB-high and NAPSB-low groups. c Correlation between NAPSB and tumor purity using the ESTIMATE algorithm. $\mathbf{d}$ Different expression of 28 tumor-associated immune cells calculated 
with the SSGSEA algorithm between NAPSB subgroups. e Correlation between NAPSB and the infiltration levels of six types of TIICs (CD8+ T cells, CD4+ T cells, NK cells, B cells, dendritic cells, and macrophages), which were calculated using six independent algorithms. $\mathbf{f}$ Correlation between NAPSB expression and the effector genes of the above immune cells. $(\mathbf{g}, \mathbf{h})$ Correlations between NAPSB and the $T$ cell inflamed score and the individual genes included in the T cell inflamed signature. I The activities of the various steps of the cancer immunity cycle in the NAPSB-high and NAPSB-low groups. ns, no significance; *, p-value < 0.05; **, p-value < 0.01; ***, p-value $<0.001 ; * \star \star *, p$-value $<0.0001$.

a

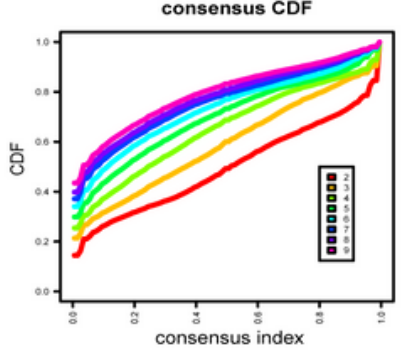

d

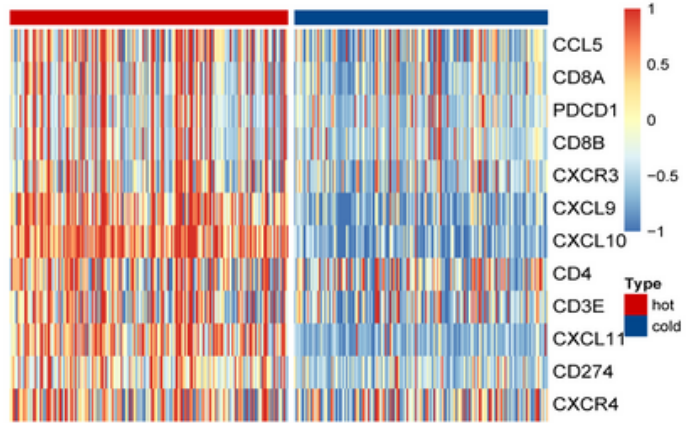

Delta area

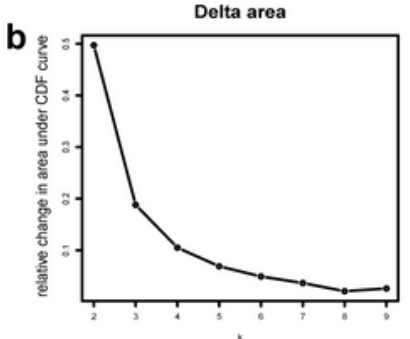

C

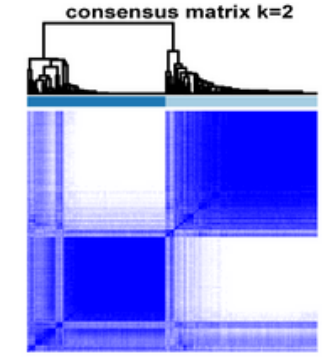

e
圆

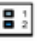

f

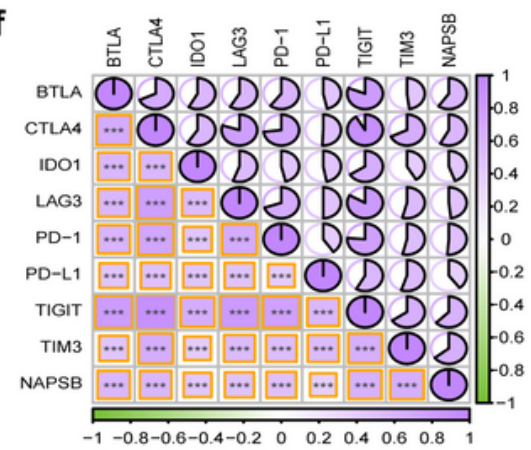

h
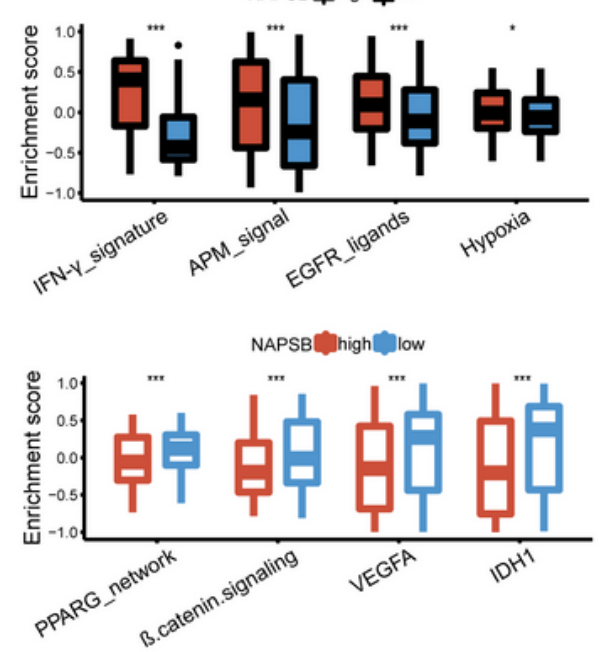

g

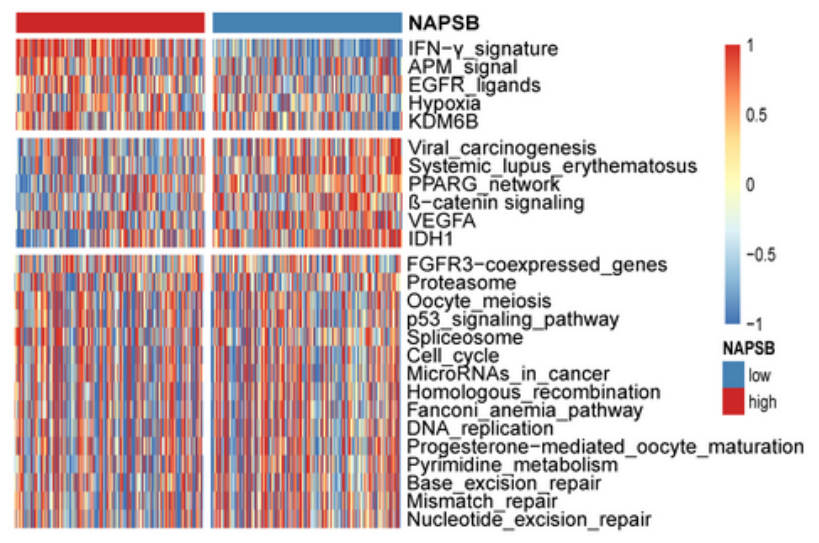

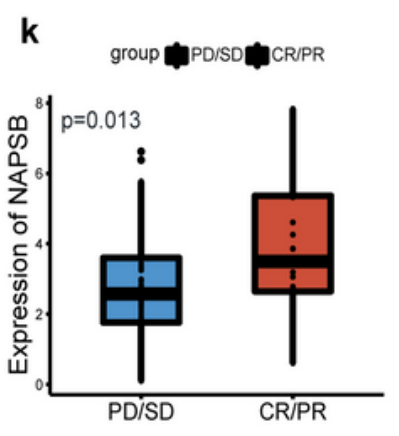




\section{Figure 4}

NAPSB was correlated with hot tumor state and improved response to immunotherapy. a Consensus clustering cumulative distribution function (CDF) for $\mathrm{k}=2-9$. $\mathbf{b}$ Relative change in area under CDF curve for $k=2-9$. c Consensus clustering heat map for $k=2$ in HCC samples. $\mathbf{d}$ Heat map plot showed hot tumor signature genes were enriched in hot tumor samples. e NAPSB was significantly overexpressed in hot tumors. $\mathbf{f}$ The expression of NAPSB was positively correlated with immune checkpoint molecules expression level. $\mathbf{g}$ Correlations between NAPSB and the enrichment scores of several therapeutic signatures. $\mathbf{h}$ Differences in enrichment scores of IFN- $\gamma$-signature, APM-signal, EGFR-ligands, hypoxia for NAPSB subgroups patients. i Differences in enrichment scores of PPARG network, $\beta$-catenin signaling pathway, VEGFA and IDH1 for NAPSB subgroups patients. $j$ The proportion of immune response to immunotherapy of NAPSB subgroups in GSE91061. $k$ NAPSB was highly expressed in CR/PR group in GSE91061. CR/PR: Complete and partial response. ns, no significance; *, p-value < 0.05; **, p-value < 0.01; $\star \star \star *, p-v a l u e<0.001 ; * \star \star \star$, p-value $<0.0001$. 

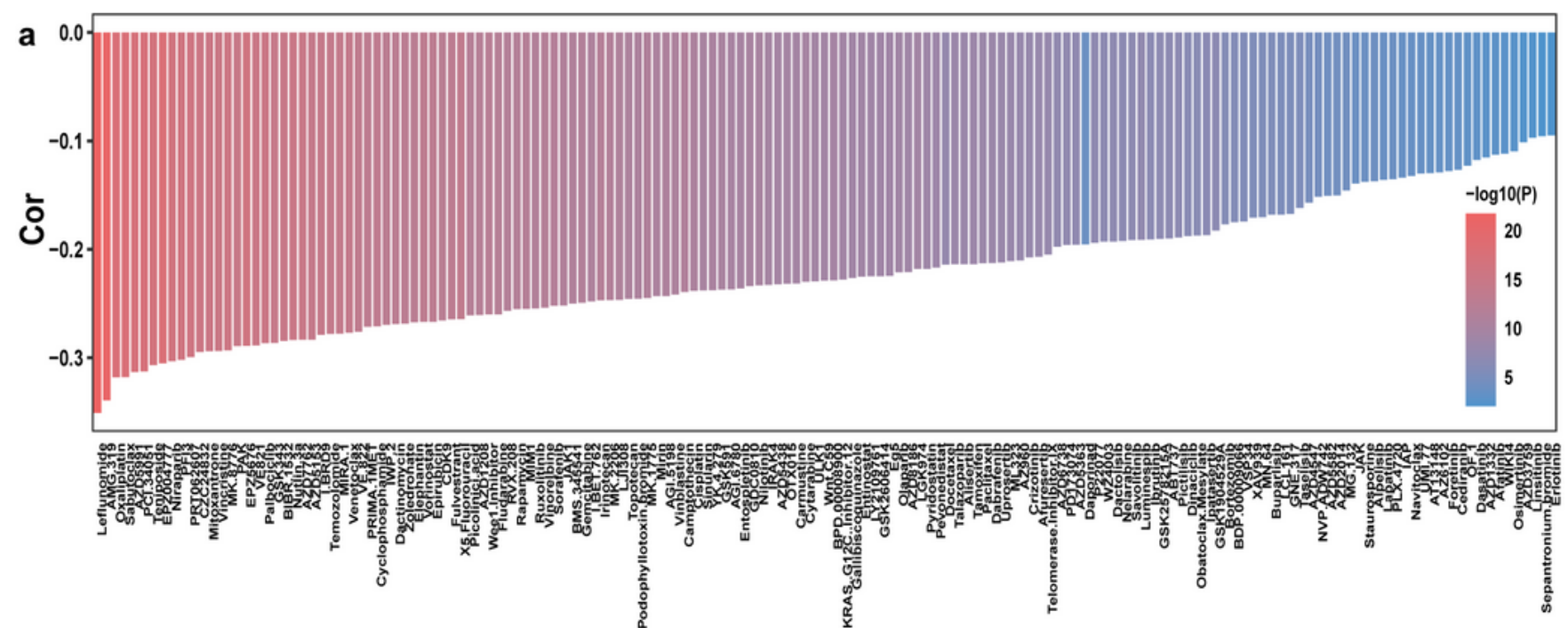

b

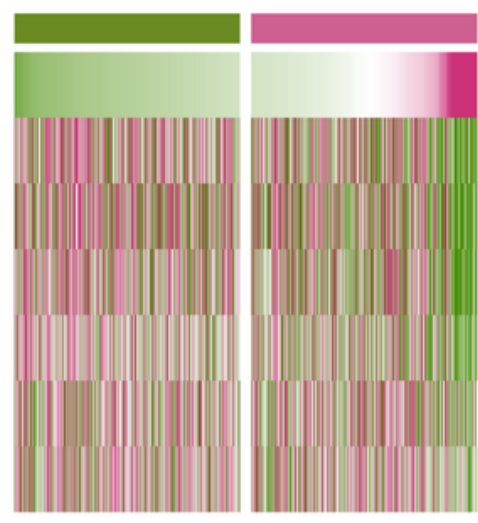

NAPSB

NAPSB

X5.Fluorouracil

Gemcitabine

Cisplatin

Sorafenib

Erlotinib

Gefitinib
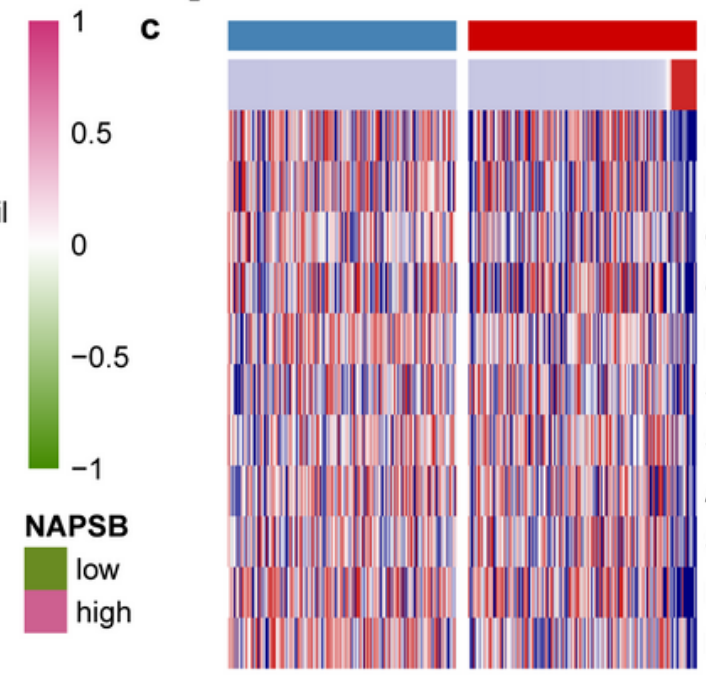

NAPSB

NAPSB

Doxorubicin

Fluorouracil

Carboplatin

Gemcitabine

Lenvatinib

Sorafenib

Sabozantinib

Axitinib

Sunitinib

Etoposide

Linifanib
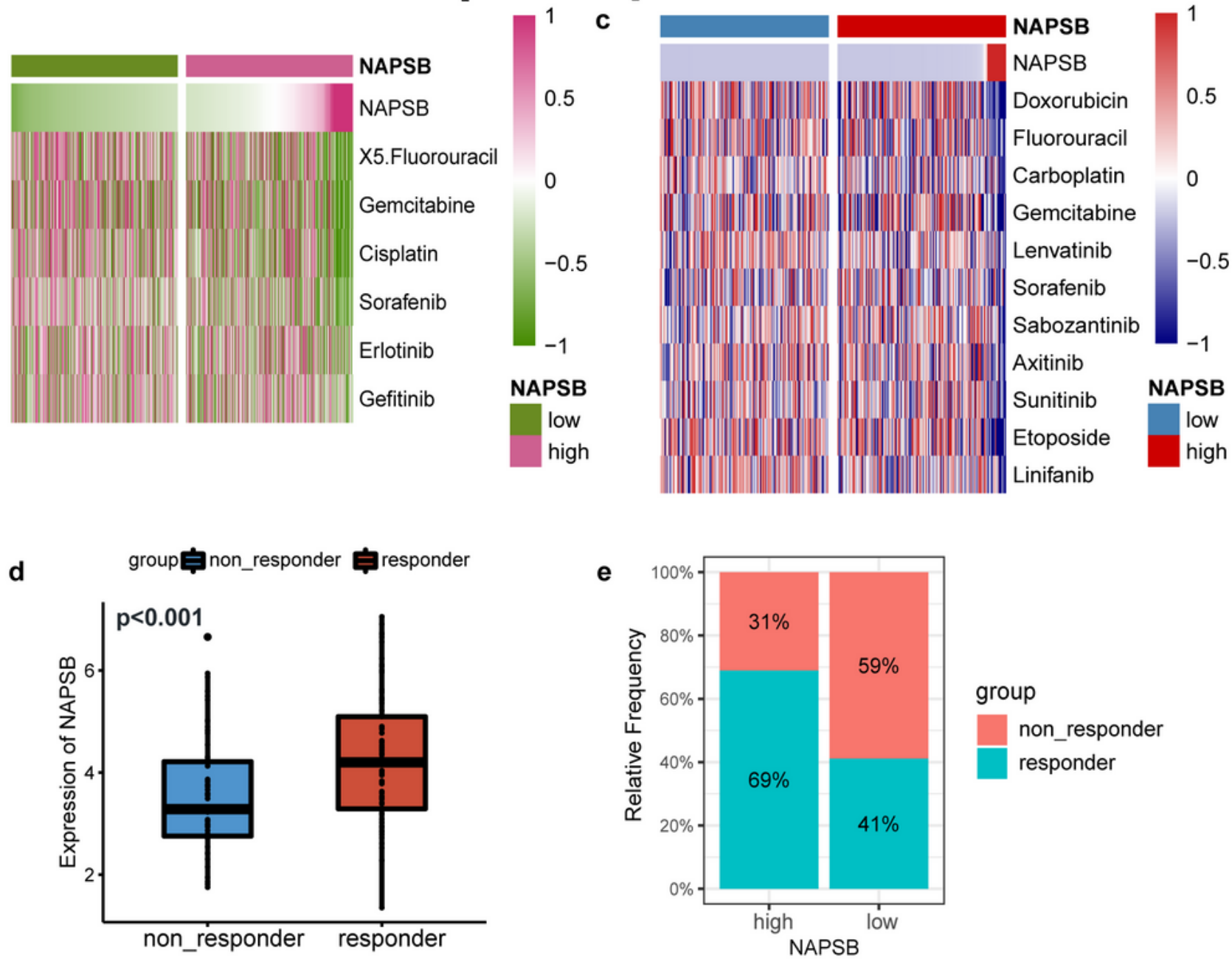

\section{Figure 5}

Potential predictive chemotherapy value of NAPSB expression. a Bar plot exhibiting Spearman correlation between NAPSB and the IC50 of drugs in GDSC. $\mathbf{b}$ Correlations between NAPSB and the IC50 of the frequently used drugs for advanced HCC patients in CTRP, including doxorubicin, fluorouracil, carboplatin, gemcitabine, lenvatinib, sorafenib, sabozantinib, axitinib, sunitinib, etoposide, and linifanib. c Correlations between NAPSB and the IC50 of the frequently used drugs for advanced HCC patients in GDSC, including 
fluorouracil, gemcitabine, cisplatin, sorafenib, erlotinib and grfitinib. d NAPSB was highly expressed in CR/PR group of transarterial chemoembolization (TACE) therapy in GSE104580. $\mathbf{e}$ The proportion of response to TACE of NAPSB subgroups in GSE104580. ns, no significance; *, p-value < 0.05; **, p-value < $0.01 ; * \star \star$, p-value $<0.001 ; * \star \star \star$, p-value $<0.0001$.
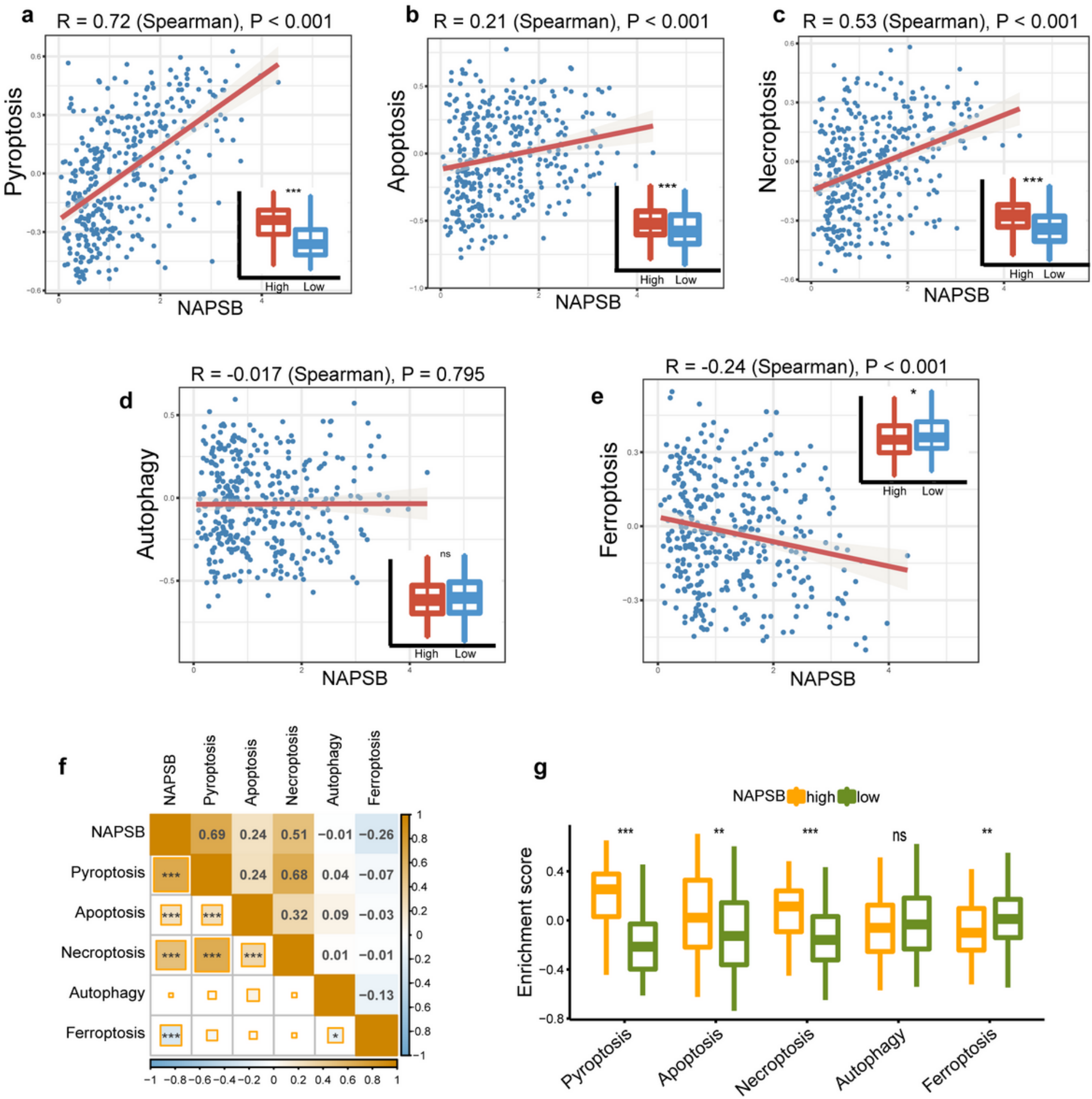

Figure 6 
Correlations between NAPSB and the enrichment scores of several cell death signatures in TCGA (a-e) and ICGC $(\mathbf{f}, \mathbf{g})$ cohort. NAPSB expression was positively correlated with pyroptosis $(\mathbf{a} ; \mathrm{R}=0.72, \mathrm{P}<$ $0.001)$, apoptosis necroptosis $(\mathbf{b} ; \mathrm{R}=0.21, \mathrm{P}<0.001)$ and necroptosis $(\mathbf{c} ; \mathrm{R}=0.53, \mathrm{P}<0.001)$. $\mathbf{d}$ Autophagy had no correlation with NAPSB expression $(R=-0.017, P=0.795)$. e NAPSB was negatively correlated with ferroptosis $(R=-0.24, P<0.001)$. $f$ Correlations between NAPSB and several forms of cell death in ICGC cohort. $\mathbf{g}$ The enrichment scores of pyroptosis, necroptosis and apoptosis in NAPSB-high groups were markedly higher than NAPSB-low groups. However, ferroptosis scores were lower in NAPSBhigh group than in NAPSB-low group and autophagy scores had no significant differences in NAPSB subgroups. Spearman coefficients were used to explore the correlations. ns, no significance; ${ }^{*}, \mathrm{p}$-value < $0.05 ; * \star$, p-value $<0.01 ; * \star \star, p$-value $<0.001 ; * \star \star \star, ~ p-v a l u e<0.0001$.

\section{Supplementary Files}

This is a list of supplementary files associated with this preprint. Click to download.

- SupplementaryFigures.zip

- SupplementaryTables.zip 